\title{
Câncer em tempos de COVID-19: repercussões na vida de mulheres em tratamento oncológico
}

\author{
Cancer in time of COVID-19: repercussions in the lives of women undergoing oncological treatment \\ Cáncer en los tiempos de COVID-19: repercusiones em la vida de las mujeres sometidas a tratamiento oncológico
}

\author{
Jeane Barros de Souza'; Vander Monteiro da Conceiçãol'; Jeferson Santos Araújo'II; \\ Júlia Valéria de Oliveira Vargas Bitencourt ${ }^{\prime v}$; Cláudio Claudino da Silva Filhov; Maíra Rossetto ${ }^{v}$
}

\begin{abstract}
RESUMO
Objetivo: compreender a vivência do enfrentamento e repercussões da COVID-19, na percepção de mulheres em tratamento oncológico. Método: estudo qualitativo, do tipo ação-participante, fundamentado no Itinerário de Pesquisa de Paulo Freire, que possui três fases: Investigação Temática; Codificação e Descodificação; Desvelamento Crítico. Foi realizado Círculo de Cultura virtual, com a participação de 12 mulheres em tratamento do câncer de mama, de diferentes localidades do Brasil. Resultados: no Círculo de Cultura virtual discutiram dois temas: desafios no enfrentamento do câncer e da COVID-19; aprendizados gerados nessa vivência, considerando um renascimento das próprias cinzas. Considerações finais: o momento pandêmico tem instigado reflexões sobre o viver. Assim, as mulheres em tratamento oncológico e também em restrição social puderam expressar seus sentimentos, descobrindo e redescobrindo fragilidades e fortalezas para ressignificar e crescer como seres, em uma sociedade, que pode e deve articular estratégias para promoção da saúde.
\end{abstract}

Descritores: Oncologia; Neoplasias da Mama; Quarentena; Infecções por Coronavírus.

\section{ABSTRACT}

Objective: to understand the experience of coping with COVID-19, as perceived by women undergoing cancer treatment. Method: qualitative, participatory action research based on the three phases of Paulo Freire's Research Itinerary: Thematic Investigation; Coding and Decoding; and Critical Unveiling. A Culture Circle was held online with 12 women from different places in Brazil undergoing breast cancer treatment. Results: in the virtual Culture Circle, they discussed two themes: challenges in coping with cancer and COVID-10; and learning generated in that experience, with a view to rebirth from their own ashes. Final considerations: the pandemic has prompted thinking about living. Accordingly, women undergoing cancer treatment and also under social restrictions were able to express their feelings, and in discovering and rediscovering weaknesses and strengths, to resignify themselves and to grow in a society that can and should deploy strategies for health promotion.

Descriptors: Medical Oncology; Breast Neoplasms; Quarantine; Coronavirus Infections.

\section{RESUMEN}

Objetivo: comprender la experiencia de afrontamiento del COVID-19, según la perciben las mujeres en tratamiento oncológico. Método: investigación-acción cualitativa y participativa basada en las tres fases del Itinerario de Investigación de Paulo Freire: Investigación Temática; Codificación y decodificación; y revelación crítica. Se realizó un Círculo Cultural en línea con 12 mujeres de diferentes lugares de Brazil sometidas a tratamiento contra el cáncer de mama. Resultados: en el Círculo de Cultura virtual se discutieron dos temas: desafíos en el afrontamiento del cáncer y COVID-10; y el aprendizaje generado en esa experiencia, con miras a renacer de sus propias cenizas. Consideraciones finales: la pandemia ha llevado a pensar en vivir. En consecuencia, las mujeres en tratamiento oncológico y también bajo restricciones sociales pudieron expresar sus sentimientos, y al descubrir y redescubrir debilidades y fortalezas, resignificarse y crecer en una sociedad que puede y debe desplegar estrategias de promoción de la salud.

Descriptores: Oncología Médica; Neoplasias de la Mama; Cuarentena; Infecciones por Coronavirus.

\section{INTRODUÇÃO}

Em dezembro de 2019, na China, identificaram-se casos recorrentes de pneumonia severa na população, o que alertou as autoridades para possível propagação de um microrganismo, identificado como Severe Acute Respiratory Syndrome Coronavirus 2 (SARS-CoV-2), o vírus causador da Coronavirus Disease 2019 (COVID-19), o qual foi transmitido comunitariamente, com disseminação mundial, alcançando o status de pandemia ${ }^{1}$. Com isso, o abrupto contágio da população exigiu das autoridades sanitárias o emprego de medidas para o controle de propagação, as quais foram: o isolamento, a quarentena, o distanciamento social e a contenção comunitária².

'Enfermeira. Doutora. Professora Adjunta. Universidade Federal da Fronteira Sul. Chapecó, Santa Catarina, Brasil. E-mail: jeane.souza@uffs.edu.br. ORCID https://orcid.org/0000-0002-0512-9765

"Enfermeiro. Doutor. Professor Adjunto. Universidade Federal da Fronteira Sul. Chapecó, Santa Catarina, Brasil. E-mail: vander.conceicao@uffs.edu.br. ORCID: https://orcid.org/0000-0003-0972-0795

I'Enfermeiro. Doutor. Professor Adjunto. Universidade Federal da Fronteira Sul. Chapecó, Santa Catarina, Brasil. E-mail: jeferson.araujo@uffs.edu.br. ORCID: https://orcid.org/0000-0003-3311-8446

IVEnfermeira. Doutora. Professora Adjunta. Universidade Federal da Fronteira Sul. Chapecó, Santa Catarina, Brasil. E-mail: julia.bitencourt@uffs.edu.br. ORCID https://orcid.org/0000-0002-3806-2288

VEnfermeiro. Doutor. Professor Adjunto. Universidade Federal da Fronteira Sul. Chapecó, Santa Catarina, Brasil. E-mail: claudio.filho@uffs.edu.br. ORCID: https://orcid.org/0000-0002-5961-9815

viEnfermeira. Doutora. Professora Adjunta. Universidade Federal da Fronteira Sul. Chapecó, Santa Catarina, Brasil. E-mail: maira.rossetto@uffs.edu.br. ORCID: https://orcid.org/0000-0002-5683-4835 
A princípio, de acordo com o levantamento epidemiológico com indivíduos infectados pelo SARS-CoV-2, identificou-se que a maioria dos casos ocorre em pessoas idosas, com comorbidades ou trabalhadores da saúde ${ }^{3}$. Assim, indivíduos em tratamento oncológico agregam ao grupo de risco, pois a história natural da doença neoplásica e seus tratamentos tendem a enfraquecer a resposta imunológica dos adoecidos, tornando-os susceptíveis a infecções respiratórias ${ }^{4}$. Portanto, a esse grupo reforça-se a manutenção das medidas contra a propagação da COVID-19. Nesse contexto, emergiu o seguinte questionamento: como é a vivência do enfrentamento e repercussões da COVID-19, na percepção de mulheres em tratamento oncológico?

É importante ressaltar que o adoecer por câncer repercute em diversas dimensões do cotidiano, como trabalho, relações sociais e conjugais, imagem corporal, sexualidade e lazer. Em tempos de pandemia, pode-se dizer que o adoecido limita sua relação com o mundo social um pouco mais do que já vivenciado. Em revisão sistemática sobre as repercussões do câncer entre profissionais de saúde, paciente e familiares, o elemento "comunicação" foi evidenciado por facilitar a translação de informações entre as pessoas e reduzir diversas barreiras criadas durante o processo de adoecimento ${ }^{5}$, como a barreira social.

Para sanar a barreira de comunicação e o distanciamento físico necessário para evitar a propagação do SARS-CoV2, pode-se considerar o emprego das tecnologias comunicativas, como o uso de softwares que realizam videochamadas, permitindo a aproximação afetuosa na restrição social ${ }^{6}$. Nesse cenário, pesquisadores com experiência no desenvolvimento e mediação do Círculo de Cultura, proposto por Paulo Freire, optaram por realizá-lo de modo virtual com mulheres em tratamento para o câncer. Assim, despontou-se este estudo com o objetivo de compreender a vivência do enfrentamento e repercussões da COVID-19, na percepção de mulheres em tratamento oncológico.

O estudo se justifica por se tratar de um público vulnerável diante da COVID-19, que carece do olhar atento dos enfermeiros e demais profissionais da área da saúde. Além disso, por meio do estudo, é possível estabelecer conexões e superar fronteiras no combate ao medo, ansiedade e outras repercussões que influenciam o processo saúde-doença das mulheres na vivência do câncer e do atual momento pandêmico.

\section{REFERENCIAL TEÓRICO}

O estudo teve como embasamento teórico os pressupostos de Paulo Freire, que busca a transformação dos sujeitos, criando relações de cuidado e de afeto por meio de uma relação ética, humanizada e de respeito ao ser humano, aos seus valores e crenças pela práxis dialógica. Somado a isso, compreende-se que todo sujeito possui seus saberes e que devem ser considerados para estabelecimento da amorosidade e troca de experiências, a fim de empoderá-los, incentivando a autonomia em busca da libertação do oprimido ${ }^{7}$. Assim, criam-se possibilidades para profissionais da saúde e pesquisadores estabelecerem relação com as mulheres no enfrentamento do câncer e da COVID-19.

\section{MÉTODO}

Trata-se de um estudo qualitativo, do tipo ação-participante ${ }^{8}$, realizado por meio do Círculo de Cultura, em que se percorreu o Itinerário de Pesquisa de Paulo Freire. A trajetória do Itinerário de Pesquisa desdobra-se de forma sistêmica em três fases, que estão interligadas entre si: 1) Investigação Temática: consiste no diálogo inicial que conduz a identificação dos temas geradores, permitindo transparecer as contradições imbricadas à vida humana em sociedade, na dinâmica de toda e qualquer realidade; 2) Codificação e Descodificação: reflexão crítica dos temas por meio de códigos que se caracterizam por serem objetos cognoscíveis para após analisar a situação vivida dialeticamente, com vistas à superação de situações limites; 3) Desvelamento Crítico: quando os participantes se permitem retirar o véu que os limita para enxergar a análise e veracidade dos fatos, desvelando o interior de suas ideias para alavancar, por meio do conhecimento, a transformação ${ }^{7}$.

No entanto, diante da situação de restrição social imposta pela COVID-19, foi conduzido o Círculo de Cultura de forma virtual, sendo algo inovador. Para tanto, utilizou-se o aplicativo Zoom ${ }^{\circledR}$, por meio de dispositivos eletrônicos (computador ou celular), viabilizando a participação interativa e simultânea das participantes, mesmo estando distantes geograficamente.

Para a organização do Círculo de Cultura Virtual (CCV), inicialmente, foram convidadas para integrar o estudo três mulheres em tratamento oncológico em um hospital público de Santa Catarina. Por meio do método de amostragem Snowball ${ }^{9}$, essas mulheres convidaram outras amigas que também passam pelo processo de câncer para compor o Círculo, assim, tendo a participação total de 12 mulheres, de diferentes localidades do Brasil. Como critérios de inclusão, foram consideradas as mulheres que estavam em tratamento do câncer de mama (radioterapia e quimioterapia) e em restrição social no período da realização do CCV. Com relação aos critérios de exclusão foram consideradas mulheres abaixo de 18 anos e sem acesso à internet e dispositivos eletrônicos no momento do CCV. 
O CCV foi desenvolvido em um único encontro, o qual foi realizado em junho de 2020, com duração de aproximadamente duas horas e meia, sendo mediado por uma enfermeira experiente na condução de Círculos de Cultura, primeira autora desse artigo, com o apoio de registro de diário de campo.

Para iniciar o CCV, foi realizado uma analogia sobre a utilização de um lenço, objeto naturalizado pelas participantes como um símbolo de suas resistências, pois assim como o lenço necessita do tecido, costura e sabedoria para amarrá-lo, a fim do seu uso ser eficiente, compreende-se que os cuidados de enfermagem também necessitam estar fixados nas particularidades das reflexões de quem vivencia os desafios do câncer e das constantes ameaças impostas pela COVID-19. Por essa perspectiva, o Itinerário de Pesquisa foi conduzido com leveza, criatividade, dialogicidade e profundidade nas etapas que o sustentam, que estiveram interligadas durante o CCV, exemplificado na Figura 1.

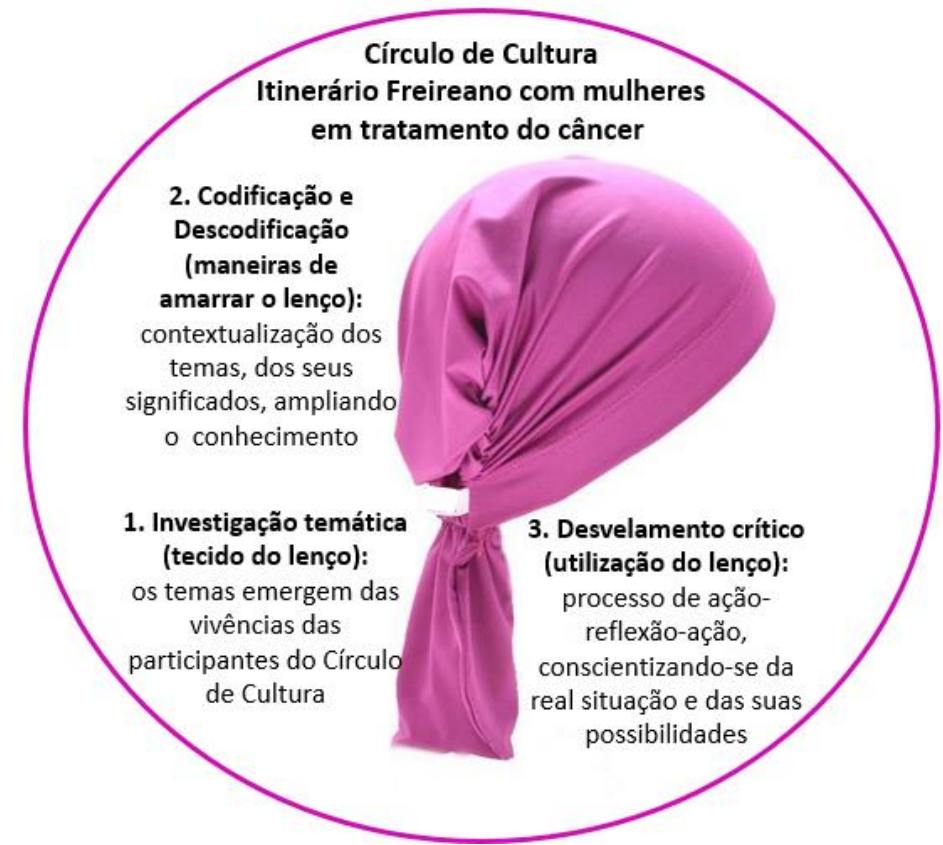

FIGURA 1: Itinerário Freireano: analogia com o uso do lenço. Brasil, 2020. Fonte: Adaptado pelos autores a partir de https://www.fitarosa.com.br/lencoquimioterapia-beanie-pink

A Investigação Temática foi construída fio a fio com todas as participantes. Para tanto, a mediadora apresentou um lenço e argumentou que se fazia necessário investigar com atenção o tecido, para obter informações sobre a durabilidade, manutenção e o manuseio ideal até ser amarrado na cabeça. Com essa analogia, convidaram-se as mulheres a investigar seus sentimentos diante do enfrentamento do câncer e da COVID-19, pois assim como o lenço necessita ser conhecido para ser utilizado, os profissionais de saúde também necessitam conhecer suas subjetividades para melhor auxiliá-las. Para estimular o diálogo, questionou-se: quais são os seus sentimentos diante da situação de vivenciar o câncer durante a pandemia da COVID-19? Após a discussão, as mulheres elegeram dois temas geradores para costurar suas experiências no CCV.

Mediou-se, então, a Codificação e Descodificação dos temas, num constante processo de ir e vir nas questões que fortalecem o tecido de suas experiências e as permitem ressignificar a vida, mesmo diante do câncer e da COVID-19. Para tanto, acompanhadas pelo som da música Peça Felicidade, de Gabriela Melim, as mulheres registraram em uma folha de papel A4 suas percepções. Em seguida, cada participante, junto com seus elementos culturais, narrou sobre sua maneira de lidar com essas questões, seus sentimentos e dúvidas frente ao desconhecido e as incertezas sobre o futuro. Nessa etapa, cada costura realizada no tecido foi fundamental para a construção de uma teia de significados presente no CCV, permitindo assim a criação de um ambiente propício para troca de saberes de maneira crítica e reflexiva entre as participantes, típico do referencial metodológico Freireano. 
Para o Desvelamento Crítico dos temas eleitos, buscou-se a conscientização do coletivo e particular de cada experiência. Logo, simbolicamente, a mediadora colocou um lenço em sua cabeça, o amarrou e compartilhou com as mulheres a reflexão de que de nada resolveria adquirir um tecido de boa qualidade, ter conhecimento sobre as diversas maneiras de amarrá-lo, se não for utilizado de forma consciente, tornando-o significativo no seu viver. Conforme a ação problematizadora desse feito, as mulheres reagiram com base nos elementos que agenciam a sua cultura e demonstraram compreender sua realidade, refletindo sobre seu viver, desvelando os limites e as suas potencialidades no enfrentamento do câncer e da COVID-19.

Como um ato final, a mediadora convidou cada participante a expressar uma frase que representasse a possível ação transformadora sentida naquele momento. Em meio as emoções e cercadas por sentimentos de empoderamento, elas apresentaram ao grupo suas percepções. Paralelamente, a mediadora retirou o seu lenço e escreveu nele com tinta de tecido cada frase compartilhada, como uma forma de representar a vivência do CCV, que foi encerrado com todas as mulheres cantando o refrão da música O que é, o que é, de Gonzaguinha.

Alguns dias após a realização do CCV, ocorreu um encontro virtual, a fim de validar os dados com as participantes do estudo. A pesquisa seguiu os preceitos éticos da Resolução 466 de 2012, sendo que o Termo de Consentimento Livre e Esclarecido foi encaminhado via e-mail, assinado e devolvido aos pesquisadores. Preservou-se o anonimato das participantes, em que cada mulher escolheu um nome de estrela para ser denominada. A pesquisa foi aprovada pelo Comitê de Ética em parecer número 4.068.387, sob o CAAE: 32239220.7.0000.5564.

\section{RESULTADOS E DISCUSSÃO}

As participantes tinham idade entre 34 e 56 anos e residiam em diferentes localidades do Brasil: cinco em Santa Catarina, três em São Paulo, duas no Paraná e duas no Rio de Janeiro. Todas estavam em tratamento por câncer de mama, sendo dez casadas, uma viúva e uma divorciada. Em relação à escolaridade: nove com ensino superior completo, uma com ensino superior incompleto e duas com ensino médio completo.

No CCV, as participantes definiram dois temas geradores: 1) Desafios: câncer e COVID-19; 2) Aprendizagem: renascendo das próprias cinzas. Nesse ínterim, o Círculo promoveu o diálogo e reflexões, proporcionando um espaço dinâmico de aprendizagem para discussão dos temas eleitos, instigando o pensamento crítico-reflexivo das mulheres.

Na etapa da Codificação e Descodificação, buscaram-se os significados dos temas geradores, em que foram compartilhados os desafios no enfrentamento do câncer e da COVID-19, na perspectiva de explorar tais situações, ficando evidente que as mulheres reconhecem o legado imposto pela vivência da pandemia e ressignificam os desafios em momentos de aprendizado, de maneira a transformar a crise em novas oportunidades de crescimento e amadurecimento pessoal, conforme se evidencia na Figura 2.

\begin{tabular}{|c|c|}
\hline 1. DESAFIOS: câncer e COVID-19 & 2. APRENDIZAGEM: renascendo das próprias cinzas \\
\hline $\begin{array}{l}\text { Estou com dificuldades para concluir o tratamento oncológico } \\
\text { (Sol) }\end{array}$ & As adversidades produzem aprendizagem (Sol) \\
\hline Tenho medo de ser contaminada (Veja) & Vivenciar momentos de dor nos torna mais fortes (Vega) \\
\hline $\begin{array}{l}\text { Eu tenho medo de sair da quarentena para fazer tratamento } \\
\text { (Sirius) }\end{array}$ & Do limão é possível fazer uma limonada (Sirius) \\
\hline Às vezes, eu penso em desistir do tratamento (Atria) & Hoje sou mais forte que ontem (Atria) \\
\hline $\begin{array}{l}\text { Estou ansiosa e preocupada com a minha saúde e também } \\
\text { dos meus familiares (Hamal) }\end{array}$ & $\begin{array}{l}\text { Câncer dentro da quarentena está sendo uma oportunidade de } \\
\text { aproveitar a família (Hamal) }\end{array}$ \\
\hline Estouo estressada com tudo a minha volta (Nunki) & $\begin{array}{l}\text { Mesmo nesses momentos de tristeza, eu consigo também } \\
\text { perceber a alegria (Nunki) }\end{array}$ \\
\hline $\begin{array}{l}\text { Fico preocuopada porque tenho que ultrapassar as barreiras } \\
\text { das cidades vizinhas para realizar meu tratamento (Shaula) }\end{array}$ & Minha família está mais unida (Shaula) \\
\hline $\begin{array}{l}\text { Eu já esotu estressada com essas notícias desagradáveis } \\
\text { (Sargas) }\end{array}$ & $\begin{array}{l}\text { O câncer e a COVID-19 sāo também uma oportunidade de se } \\
\text { achegar mais a Deus (Sargas) }\end{array}$ \\
\hline Sinto tristeza e ansiedade (Polaris) & Eu tenho esperança de dias melhores (Polaris) \\
\hline Por que tudo isso agora? Já não bastava o câncer? (Capella) & As lutas da vida desenvolvem a fé e a esperança (Capella) \\
\hline Eu tenho preocupação com o amanhã (Castor) & $\begin{array}{l}\text { Tudo isso é oportunidade de crescimento e amadurecimento } \\
\text { (Castor) }\end{array}$ \\
\hline Será que sairemos vitoriosas dessas lutas? (Antares) & Sou uma nova mulher (Antares) \\
\hline
\end{tabular}

FIGURA 2: Desafios e aprendizagem no enfrentamento do câncer e da COVID-19. Brasil, 2020.

Fonte: Elaborado pelos autores. 
Por certo, frente aos desafios desvelados, é possível evidenciar que a pandemia por COVID-19 estabelece barreiras para a continuidade do tratamento oncológico, pois fomenta um ambiente não harmonioso na vida das mulheres. Além disso, pode gerar conflitos que, caso não assistidas de forma adequada pela equipe de enfermagem, repercutirão na omissão cuidado. Por outro lado, os sentimentos como medo, tristeza, stress e preocupação se fazem presentes, o que pode aumentar a ansiedade e a incerteza quanto à cura, tornando-as mais vulneráveis.

Em suma, o câncer e seus tratamentos promovem transformações na vida de suas adoecidas, colocando-as em situações indesejadas para manutenção do seu corpo, além da relação familiar, trabalho e convívio social ${ }^{10}$. Isso se dá devido ao seu estigma, índice de recidiva e morte em todo mundo, sendo que a doença também é responsável por desencadear sentimentos de incerteza quanto ao futuro, o que dificulta a adesão a tratamentos prolongados e agressivos como seções de radioterapia e quimioterapia ${ }^{11}$. Somado aos agravantes naturais impostos pela doença, o contexto pandêmico desencadeado pela COVID-19 endossa esse cenário, ao gerar preocupações, ansiedade e restrição social, devido às medidas protecionistas de controle.

Ademais, a adesão ao isolamento social relaciona-se com o medo de se infectar ou de sofrer injúrias à saúde, todavia, quando não conduzido de forma harmônica, torna-se um preditor de risco para questões psicológicas, desencadeando stress, ansiedade, raiva, tédio, frustração e sensação de isolamento em relação ao resto do mundo ${ }^{12}$. Por mais que a restrição social em tempos de pandemia seja indispensável para tomada de medidas de prevenção não farmacológicas, também se faz necessária a organização de estratégias para estimular a continuidade ao tratamento oncológico, como parte de um planejamento para o fortalecimento do engajamento das mulheres.

Em tempos de ameaça à vida, como durante uma pandemia, o apoio dos pares torna-se uma estratégia para assistir esse público. Nesse âmbito, as pesquisas do tipo ação-participante são exitosas, ao articular aprendizado mútuo, de baixo custo, fortalecendo o engajamento das mulheres e empoeirando-as de saberes afirmativos. Os enfermeiros oncológicos também devem inserir-se nesse contexto, reconhecendo o manejo das incertezas entre os indivíduos diagnosticados com câncer e incentivando medidas educativas e de enfrentamento saudáveis ${ }^{13}$.

Com relação aos desafios impostos ao lidar com as mazelas do câncer e as inúmeras barreiras e ameaças que acompanham a pandemia, foi evidenciado que as mulheres aprendem com as adversidades e ressignificam valores para contemplar o momento como uma fonte de novas oportunidades, ancorando-se no sentimento de esperança e força que precisam para sobreviver frente a tudo isso.

Sendo assim, a esperança é um elo positivo na condução psicológica daquilo que nesse momento não é possível, mas que por meio da projeção de um futuro promissor algum dia poderá ser ${ }^{14}$. Essa característica fortalece comportamentos no presente, em busca por estratégias de enfrentamento e de resistência, que são elementos essenciais para o engajamento dessas mulheres nos tratamentos, por meio das medidas de prevenção e cura nesse momento de pandemia.

Dessa forma, as mulheres puderam compreender sua realidade, refletir sobre seu viver, desvelando seus limites e suas potencialidades no enfrentamento do câncer e da COVID-19. Ademais, as participantes revelaram ter apreciado a participação no CCV, demonstrando gratidão, aprendizado, acolhimento e transformação mútua, como ilustra a Figura 3.

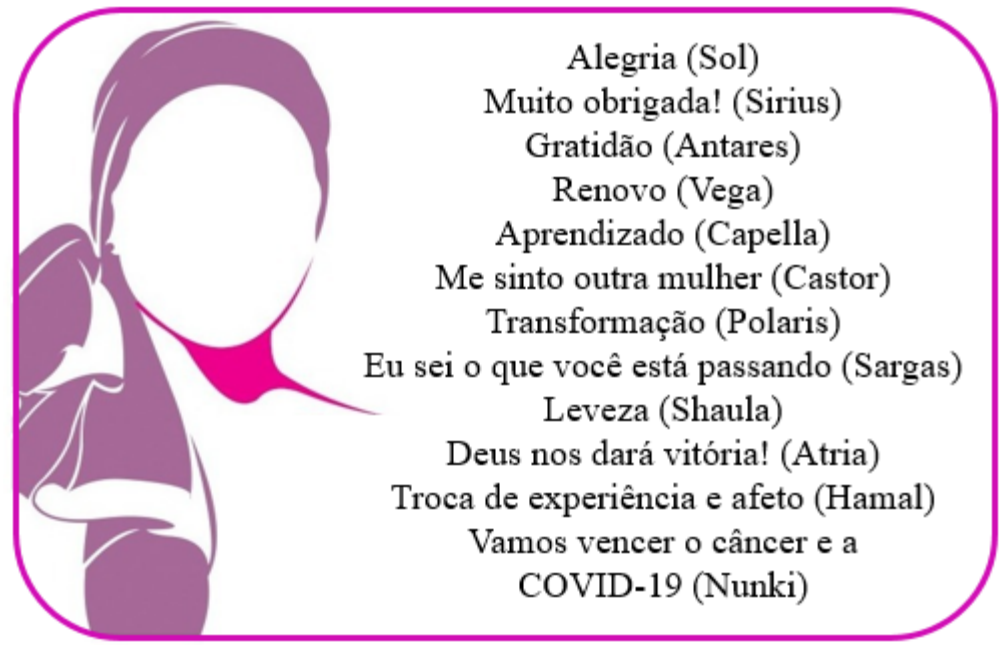

FIGURA 3: Vivência no Círculo de Cultura Virtual. Brasil, 2020.

Fonte: Adaptado pelos autores, a partir de https://www.fitarosa.com.br/lencoquimioterapia-beanie-pink 
As mulheres demonstraram gratidão e felicidade, cantando juntas e com empolgação, ficando evidente que durante a atividade elas se transformaram, saindo diferentes da maneira que ingressaram. Além disso, diante de tantas inquietações sobre a vivência do câncer e do distanciamento social imposto pela COVID-19, a utilização da música contribuiu para a promoção da saúde, proporcionando momentos de redução do stress, diversão, reflexão das mensagens partilhadas e estabelecimento de vínculo ${ }^{15}$ entre as participantes.

Outrossim, a disponibilização de espaços para o cuidado on-line também se apresentou como uma ferramenta de apoio. Na situação de restrição social e vivência de um câncer, torna-se necessário promover espaços de comunicação, com vistas a sanar dúvidas, promover acolhimento e apoio emocional. O cuidado on-line é uma estratégia já conhecida no setor saúde, mas durante a pandemia COVID-19 tornou-se popular entre os brasileiros, sendo que enfermeiros e outros profissionais apresentam-se cada vez mais engajados nessa modalidade, fornecendo orientações por meio de dispositivos móveis, redes socias e aplicativos específicos ${ }^{16}$.

No atual panorama da saúde, vivencia-se um período em que práticas exitosas promotoras de cuidado ganham espaços frente aos meios tradicionais, com inserção de novas terminologias de saúde e alteração de rotinas $^{17}$. No caso, adaptar-se ao novo torna-se um convite para a sobrevivência. Nessa perspectiva, os cuidados oncológicos também necessitam ser planejados, sendo que questões como as evidenciadas pelas depoentes nesse estudo tornam-se importantes indicadores para a fidelização dessas aos tratamentos.

Diante da imposição necessária da restrição social, como forma de controle da transmissibilidade do SARS-CoV-2, a habilidade humana de se reinventar foi acionada. Assim, com os recursos tecnológicos da atualidade, tem sido possível aproximar pessoas sem aproximar corpos. Por meio do CCV foi oportunizado diálogo, troca de saberes, em um momento em que a restrição social atravessava a vida das mulheres que experienciavam o câncer de mama, impondo a elas maiores demandas psicológicas, econômicas e sociais. Assim, foi possível promover saúde em um contexto histórico inimaginável e dialeticamente desafiador.

Nesse cenário, o CCV se desvela como uma ferramenta para a Enfermagem desenvolver pesquisas e também promover a saúde em tempos de COVID-19, possibilitando acolhimento humanizado e integral, mesmo não sendo de forma presencial, principalmente aos grupos mais vulneráveis, como no caso das pessoas que possuem comorbidades, tal qual na situação das mulheres que vivenciam o câncer.

Como limitação do estudo, cita-se a abrangência de mulheres que participaram, pois é fato que uma organização dessa natureza não deve estar composta por um grupo maior de pessoas, para que não se impeça a efetiva participação, bem como a fluidez de ideias. Mas ao mesmo tempo em que se reconhece essa limitação, que é inerente à opção metodológica, se entende que seja factível a replicação dessa proposição a outros públicos, em outras localidades do Brasil e mundo, a fim de além da pesquisa também viabilizar espaço de discussão e promoção da saúde por meio do CCV.

\section{CONSIDERAÇÕES FINAIS}

O momento pandêmico atual tem instigado reflexões sobre inúmeros aspectos da vida humana, desde questões cotidianas e práticas do dia a dia até situações que remetem aos sentidos da vida. Assim, foi em meio a esse cenário de intensos chamamentos ao pensar que o estudo permitiu de maneira simultânea que mulheres que experienciam o tratamento oncológico e também a restrição social pudessem expressar seus sentimentos, olhando para si e para suas parceiras de vivências, descobrindo e redescobrindo fragilidades e forças para que, por meio da troca de saberes, se ressignificassem, crescendo como seres em uma sociedade que pode e deve se articular para promover saúde.

Não obstante, com a impossibilidade de encontros presenciais devido à COVID-19, despontou o ensejo para os profissionais da saúde realizarem escuta qualificada por meio do CVV, oportunizando trocas e aprendizado mútuo. Nesse contexto, o CCV pautado no referencial de Paulo Freire emerge como uma ferramenta metodológica e assistencial para a Enfermagem, possibilitando o desenvolvimento de cuidado humano, integral, acolhedor, promovendo o empoderamento e a autonomia dos sujeitos participantes.

\section{REFERÊNCIAS}

1. Carico RR, Sheppard J, Thomas CB. Community pharmacists and communication in the time of COVID-19: Applying the health belief model. Research in Social and Administrative Pharmacy [Internet]. 2020 [cited 2020 Jul 13]; 25:1-4. DOI: https://doi.org/10.1016/j.sapharm.2020.03.017

2. Jin H, Lu L, Liu J, Cui M. Complex emergencies of COVID-19: management and experience in Zhuhai, China. International Journal of Antimicrobial Agents [Internet]. 2020 [cited 2020 Jul 13]; 28:1-2. DOI: https://doi.org/10.1016/j.ijantimicag.2020.105961

3. Oliveira ES, Morais ACN. COVID-19: uma pandemia que alerta à população. InterAm. J. Med. Healt [Internet]. 2020 [cited 2020 Jul 13]; 2:1-4. DOI: https://doi.org/10.31005/iajmh.v3i0.80

4. Thuler LCS, Melo AC. SARS-CoV-2/COVID-19 in Patients with Cancer. Rev. Brasileira Cancerologia [Internet]. 2020 [cited 2020 jul 13]; 66(2):1-2. DOI: https://doi.org/10.32635/2176-9745.RBC.2020v66n2.970 
5. Bennardi M, Diviani N, Gamondi C, Stüssi G, SalettiP, Cinesi I, Rubinelli S. Palliative care utilization in oncology and hematooncology: a systematic review of cognitive barriers and facilitators from the perspective of healthcare professionals, adult patients, and their families. BMC palliat. care [Internet]. 2020 [cited 2020 Jul 13]; 19(47):1-17. DOI: https://doi.org/10.1186/s12904-020-00556-7

6. Nekhlyudov L, Duijts S, Hudson SV, Jones JM, Keogh J, Love B, Lustberg M, Smith KC, Tevaarwerk A, Yu X, Feuerstein M. Addressing the needs of cancer survivors during the COVID-19 pandemic. Journal of Cancer Survivorship [Internet]. 2020 [cited 2020 Jul 13]; 25:1-6. DOI: https://doi.org/10.1007/s11764-020-00884-w

7. Heidemann ITSB, Dalmolin IS, Rumor PCF, Cypriano CC, Costa MFBNA, Durand MK. Reflections on paulo freire's research itinerary: contributions to health. Rev. Texto \& Contexto Enfermagem [Internet]. 2017 [cited 2020 Jul 13]; 26(4). DOI: https://doi.org/10.1590/0104-07072017000680017

8. Felcher CDO, Ferreira ALA, Folmer V. From action-research to participant research: discussions from an investigation developed on the Facebook. Rev. Experiências em Ensino de Ciências. [Internet]. 2017 [cited 2020 Jul 13]; 12(7):1-18. Available from: http://if.ufmt.br/eenci/artigos/Artigo_ID419/v12_n7_a2017.pdf

9. Ghaljaie F, Naderifar M, Goli, H. Snowball Sampling: A Purposeful Method of Sampling in Qualitative Research. Strides in Development of Medical Education [Internet]. 2017 [cited 2020 Jul 13]; 14(3). Available from: http://sdme.kmu.ac.ir/article_90598.html

10. Araújo JS, Conceição VM, Zago MMF. Transitory masculinities in the context of being sick with prostate cancer. Rev. LatinoAmericana de Enfermagem [Internet]. 2019 [cited 2020 Jul 13]; 27: e3224. DOI: http://dx.doi.org/10.1590/15188345.3248 .3224

11. Zhang Y. Uncertainty in illness: theory review, application, and extension. Oncology Nursing Forum [Internet]. 2017 [cited 2020 Jul 13]; 44(6):645-9. DOI: http://dx.doi.org/10.1188/17.0NF.645-649

12. Bezerra ACV; Silva CEM: Soares FRG; Silva JAM. Factors associated with people's behavior in social isolation during the COVID19 pandemic. Rev. Ciência \& Saúde Coletiva [Internet]. 2020 [cited 2020 Jul 13]; 11:2411-21. DOI: https://doi.org/10.1590/1413-81232020256.1.10792020

13. Silva IBN, Patrício ACFAP, Leite MAP, Santos TD, Ferreira MAM, Silva RAR. Factors associated with people's behavior in social isolation during the COVID-19 pandemic. R. pesq.: cuid. fundam. online. 2020 [cited $2020 \mathrm{Jul}$ 13]; 12:118-23. Available from: http://seer.unirio.br/index.php/cuidadofundamental/article/view/7103/pdf_1

14. Souza JB, Martins EL, Xirello T, Urio A, Barbosa SSP, Pitilin EB. Interface between music and women's health promotion. Rev. Brasileira Promoção da Saúde [Internet]. 2020 [cited 2020 Jul 13]; 33:9466. Available from: https://periodicos.unifor.br/RBPS/article/view/9466

15. Oliveira WK; Duarte E; França GVA; Garcia LP. How Brazil can hold back COVID-19. Epidemiol. Serv. Saúde [Internet]. 2020 [cited 2020 jul 13]; 2(29):e2020044. DOI: https://doi.org/10.5123/s1679-49742020000200023

16. Moll MF, Boff NN, Silva PS, Siqueira TV, Ventura CAA. The family health strategy nurse and health promotion and disease prevention. Enferm. Foco [Internet]. 2019 [cited 2020 Jul 13]; 10(3):134-40. DOI: https://doi.org/10.21675/2357707X.2019.v10.n3.2001

17. Carr E. New Normal Terminology. Clinical Journal of Oncology Nursing [Internet]. 2020 [cited 2020 Jul 13], 24(3). DOI: https://doi.org/10.1188/20.CJON.223 